\title{
Laparoscopy-assisted subtotal gastrectomy with very small remnant stomach: a novel surgical procedure for selected early gastric cancer in the upper stomach
}

\author{
Xiaohua Jiang $\cdot$ Naoki Hiki $\cdot$ Souya Nunobe $\cdot$ \\ Kyoko Nohara · Koshi Kumagai · Takeshi Sano • \\ Toshiharu Yamaguchi
}

Received: 7 July 2010/Accepted: 1 December 2010/Published online: 24 February 2011

(C) The International Gastric Cancer Association and The Japanese Gastric Cancer Association 2011

\begin{abstract}
Total gastrectomy or proximal gastrectomy is usually performed either as an open procedure or laparoscopically for the treatment of early gastric cancer (EGC) in the upper stomach. However, quality of life after either total or proximal gastrectomy is not so satisfactory. The authors report a novel surgical procedure, laparoscopyassisted subtotal gastrectomy (LAsTG), by which a very small remnant stomach is preserved, for the surgery of selected EGCs in the upper stomach. Twenty-three patients with EGC in the upper stomach underwent LAsTG. After lymph node dissection and mobilization of the stomach, the stomach was transected about $2 \mathrm{~cm}$ proximal to the tumor and a very small remnant stomach was preserved. An anvil was inserted transorally into the remnant stomach by using the OrVil ${ }^{\mathrm{TM}}$ system. The reconstruction method was Rouxen-Y, and hemidouble-stapling gastrojejunostomy with a circular stapler was performed intracorporeally. There were no intraoperative complications or conversions to open surgery. Mean operation time and blood loss were $266.7 \mathrm{~min}$ and $54.6 \mathrm{ml}$, respectively. The overall incidence of early postoperative complications was $17.4 \%$, and two patients underwent reoperation because of duodenal stump leakage and stenosis of the Y-anastomosis, respectively. During the follow-up period, two patients experienced
\end{abstract}

\footnotetext{
X. Jiang $\cdot$ N. Hiki $(\bowtie) \cdot$ S. Nunobe

K. Nohara $\cdot$ K. Kumagai · T. Sano $\cdot$ T. Yamaguchi

Department of Gastroenterological Surgery,

Gastroenterological Center, Cancer Institute Hospital,

Japanese Foundation for Cancer Research,

3-8-31 Ariake, Koto-ku, Tokyo 135-8550, Japan

e-mail: naoki.hiki@jfcr.or.jp

X. Jiang

Department of General Surgery, Zhongda Hospital,

Southeast University, Nanjing, China
}

gastrojejunostomy stenosis and both were treated successfully by endoscopic balloon dilation. LAsTG may be performed in selected patients with EGC in the upper stomach. With the described method, a very small remnant stomach can be preserved.

Keywords Laparoscopic surgery · Gastrectomy · Anastomosis · Roux-en-Y · Stomach neoplasms

\section{Introduction}

The incidence of early gastric cancer (EGC) is increasing in Japan, and EGC now accounts for more than 50\% of all gastric cancers [1]. Laparoscopic or laparoscopy-assisted gastrectomy (LAG) is now well accepted and widely used as a minimally invasive surgical treatment for EGC. Many studies have demonstrated the safety and advantage of LAG over conventional open gastrectomy [2-4]. Laparoscopy-assisted distal gastrectomy (LADG) is the most commonly used laparoscopic surgery because most EGCs are located in the middle or lower third of the stomach.

On the other hand, for the treatment of EGCs in the upper third of the stomach, the advantage of laparoscopic surgery still remains controversial. Laparoscopy-assisted proximal gastrectomy (LAPG) has been performed in some centers that are experienced with the technique of LADG $[5,6]$. However, compared to total gastrectomy, proximal gastrectomy was found to be associated with a high rate of complications such as anastomotic stenosis and reflux esophagitis, and it appears to offer no benefit in regard to weight loss [7]. Laparoscopy-assisted total gastrectomy (LATG) is another choice for EGC in the upper third of stomach. However, it has not become as popular as LADG because of its technical difficulty and because of concerns 
regarding postoperative complications [8, 9]. Lee and colleagues [10] compared the surgical outcomes of LATG with those of LADG and found that the postoperative complication rate of LATG was higher than that of LADG, especially that of anastomotic stricture.

In some patients with EGC in the upper third of the stomach, there is still a certain distance from the tumor to the esophagogastric junction (EGJ). In these selected cases, it is theoretically possible to preserve a small volume of remnant proximal stomach, which is beneficial for postoperative quality of life. However, if the remnant stomach is too small, it would be difficult to perform a laparoscopic Roux-en-Y reconstruction through a minilaparotomy or using an endoscopic linear stapler [11]. Herein we report a novel surgical procedure, laparoscopy-assisted subtotal gastrectomy (LAsTG) with a very small remnant stomach. A very small remnant proximal stomach was preserved and a Roux-en-Y anastomosis was performed by using an $\mathrm{OrVil}^{\mathrm{TM}}$ system and a circular stapler. The surgical procedures are described and the short-term surgical outcomes are reported.

\section{Patients and methods}

\section{Patients and indications}

From January 2009 to June 2010, laparoscopy-assisted gastrectomy was performed in 413 patients with preoperatively diagnosed EGC at the Department of Gastroenterological Surgery at The Cancer Institute Hospital, Tokyo, Japan. The diagnoses of tumor depth and lymph node involvement were based on the results of gastric endoscopy, barium radiography, computed tomography (CT), or endoscopic ultrasonography (EUS). LAsTG was performed in 23 patients with EGC by two experienced laparoscopic surgeons (N. Hiki and S. Nunobe). The indications for LAsTG were as follows: (1) EGC preoperatively diagnosed as T1N0; (2) tumor located in the upper third of the stomach or the tumor lesion involved the upper third of the stomach; (3) there was still a certain distance from tumor to the EGJ but the distance was $<5 \mathrm{~cm}$; and (4) the remnant gastric stump was $2-3 \mathrm{~cm}$ from the EGJ. For patients who would receive LAsTG, an extra preoperative gastroscopy was performed; the proximal margin (about $2 \mathrm{~cm}$ to the tumor) was marked by clips and was confirmed to be tumor-negative by biopsy. If the biopsy was positive for tumor, LATG, instead of LAsTG, would be performed.

Surgical technique

The patient was placed in a supine position with legs apart. The initial trocar port was placed via a $2 \mathrm{~cm}$ intraumbilical

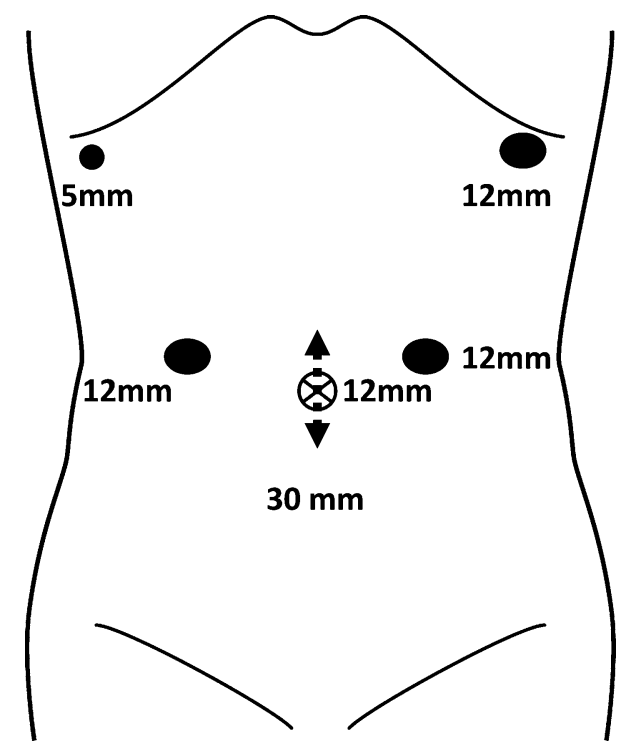

Fig. 1 Locations of the five ports. The intraumbilical port is used for the insertion of a laparoscope. Two ports are added to the right and left abdomen, respectively. The intraumbilical port is extended to $3 \mathrm{~cm}$ for a minilaparotomy

incision made by the open method. After the establishment of pneumoperitoneum at $10 \mathrm{mmHg}$, a $30^{\circ}$ laparoscope was introduced through the intraumbilical port. Another four trocars were inserted under laparoscopic vision (Fig. 1). Mobilization of the stomach and modified D2 (D1 + $7+$ $8 \mathrm{a}+9+11 \mathrm{p}$ or D2-10) lymphadnectomy were performed as previously described [12]. To decrease the incidence of gallstone formation [13], the hepatic branch of the vagus nerve was preserved routinely and the celiac branch of the vagus nerve was preserved if possible. The duodenum was divided with an endoscopic linear stapler, and an interrupted seromuscular suture was performed to prevent duodenal stump rupture.

\section{Transection of the stomach}

The left gastroepiploic vessels were divided from the root, and the lymph nodes of station $4 \mathrm{sb}$ were totally dissected. Several distal branches of the short gastric artery were also divided and at least one to two branches of the short gastric artery near the gastric cardia were preserved. The posterior gastric artery was not necessarily preserved. So, finally, the blood supply of the remnant stomach would be from the left inferior phrenic artery and one or two branches of the short gastric artery.

After stomach mobilization and lymph node dissection, intraoperative gastroscopy was performed to confirm the location of the tumor and the preoperatively placed clips, and a safe gastric transection line was determined and marked by dyeing or suturing in the outer gastric wall (Fig. 2a). Under gastroscopic direct vision, the stomach 
Fig. 2 Transection of the stomach. a The location of the tumor is confirmed by intraoperative gastroscopy, and a safe gastric transection line is marked by suturing in the outer gastric wall. b Transection for tumor in or near the lesser curvature. c Transection for tumor in the anterior or posterior gastric wall. d, e Transection for tumor in or near the greater curvature. after transection
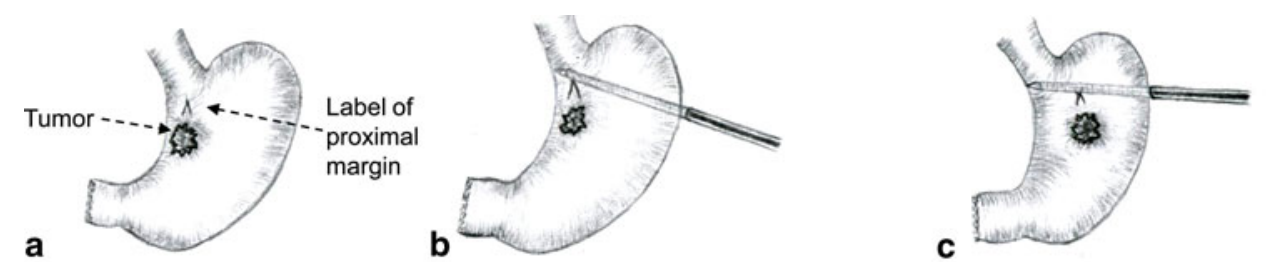
f Very small remnant stomach

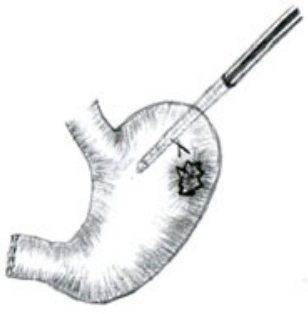

d

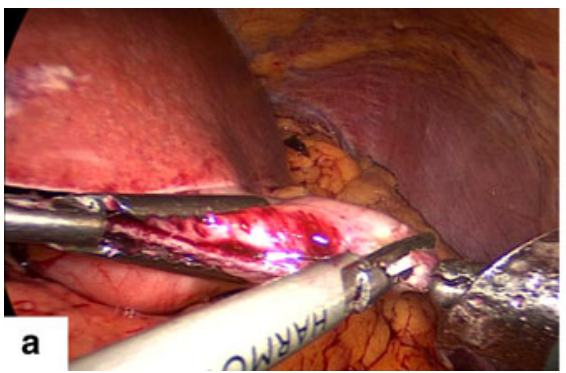

Fig. 3 Anvil insertion using the OrVil ${ }^{\mathrm{TM}}$ system. a As the OrVil ${ }^{\mathrm{TM}}$ tube reaches the greater curvature of the gastric stump, a small hole is created on the greater curvature of the gastric stump. b The OrVil ${ }^{\mathrm{TM}}$

was transected with two endoscopic linear staplers about $2 \mathrm{~cm}$ proximal to the tumor. The transection lines differed depending on the location of the tumor (Fig. 2b-e). If the tumor was in or near the lesser curvature, it would be possible to preserve more of the greater curvature (Fig. 2b); and if the tumor was in or near the greater curvature, the transection line would be higher and less of the greater curvature would be preserved (Fig. 2d, e). During transection, it is important to pay attention so that the EGJ is not transected. The remnant stomach was very small after transection (Fig. 2f).

Roux-en-Y reconstruction using the OrVil ${ }^{\mathrm{TM}}$ system and a circular stapler

After the gastrectomy, an OrVil ${ }^{\mathrm{TM}}$ tube (Covidien, Mansfield, MA, USA) with a $25-\mathrm{mm}$ anvil was introduced into the remnant stomach transorally. As the $\mathrm{OrVil}^{\mathrm{TM}}$ tube reached the greater curvature of the gastric stump, a small hole was created on the greater curvature of the gastric stump (Fig. 3a). The OrVil ${ }^{\mathrm{TM}}$ tube was extracted through the hole until the $25-\mathrm{mm}$ anvil reached the greater curvature of the gastric stump (Fig. 3b), and the tube was disconnected and pulled out from the abdomen by cutting the tube is extracted through the hole until the anvil reaches the greater curvature. c Illustration of appearance after completion of the transoral anvil insertion

thread connecting the anvil and the tube (Fig. 3c). The jejunum $20 \mathrm{~cm}$ distal to the Treitz ligament was transected using an endoscopic linear stapler and prepared as a Roux limb.

A $3 \mathrm{~cm}$ longitudinal minilaparotomy incision was then made by extending the intraumbilical port (Fig. 1). The minilaparotomy wound was retracted and protected, using a laparotomy wound retractor (Alexis Wound Retractor; Applied Medical, CA, USA), and the specimen was removed through the minilaparotomy. Lymph nodes of station $4 \mathrm{sa}, 4 \mathrm{sb}$, and the second tier (station $7,8 \mathrm{a}$, or 9) were sent for frozen pathology. The proximal margin was also sent for frozen pathology when necessary. If lymph nodes were cancer-positive, standard D2 open total gastrectomy would be performed.

Side-to-side jejunojejunostomy (Y-anastomosis), using an endoscopic linear stapler, was performed extracorporeally to create a 40-cm Roux-en-Y limb. A 25-mm circular stapler was inserted into the distal limb of the jejunum and the jejunum was tied to the stapler with a rubber band to prevent slippage of the jejunum from the circular stapler during anastomosis. The circular stapler was introduced into the abdominal cavity, and then the wound retractor and the circular stapler were sealed off, using a surgical glove, 
Fig. 4 Roux-en-Y reconstruction procedure. a The anvil and circular stapler are connected. b Anastomosis is performed laparoscopically using a hemidouble-stapling technique. c The jejunal stump is closed with an endoscopic linear stapler. d The antecolic Roux-en-Y reconstruction is completed
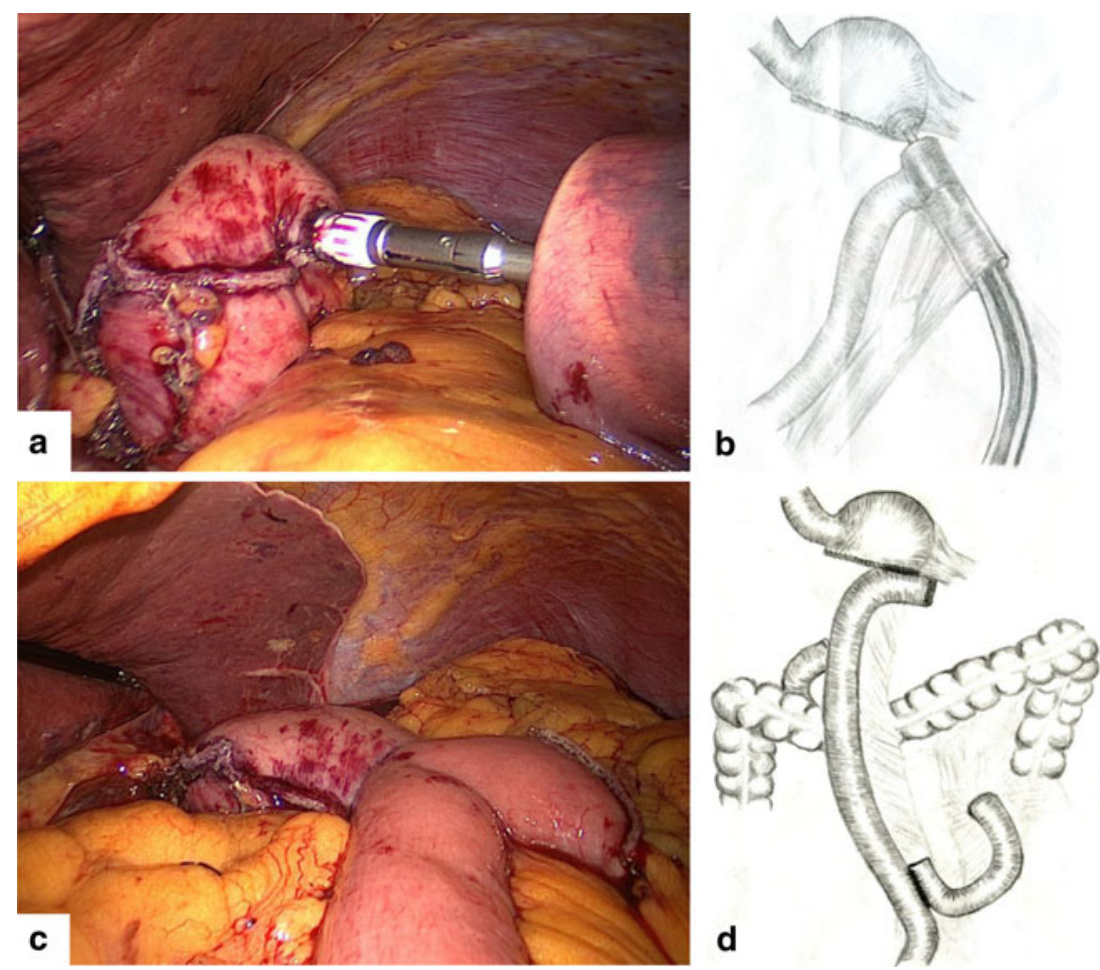

so that pneumoperitoneum could be reestablished. The port located at the right lower side of the abdomen was used for laparoscopy. The Roux-en-Y limb was then brought up via the antecolic route to create a gastrojejunostomy (Fig. 4). The anvil and circular stapler were connected (Fig. 4a), and anastomosis was performed laparoscopically, using a hemidouble-stapling technique (Fig. 4b). The jejunal stump was closed with an endoscopic linear stapler (Fig. 4c) and the antecolic Roux-en-Y reconstruction was completed (Fig. 4d). Finally, the gastrojejunal anastomosis was checked under gastroscopy to confirm that there was no bleeding, no stenosis, and no leakage. Abdominal irrigation was performed using $2000 \mathrm{ml}$ of normal saline. A silicon drainage tube was placed through the right upper port site around the gastrojejunal anastomosis. The operation was completed by closure of all the wounds.

\section{Results}

From January 2009 to June 2010, LAsTG was performed in 23 patients with EGC in the upper stomach. Of these patients, 17 were men and 6 were women; the mean age $( \pm \mathrm{SD})$ was $61.5 \pm 7.8$ years (range $38-75$ years) and mean body mass index $( \pm \mathrm{SD})$ was $23.0 \pm 2.8 \mathrm{~kg} / \mathrm{m}^{2}$. All patients were diagnosed as having stage IA gastric cancer according to the Japanese classification of gastric carcinoma (JCGC) [14]. The mean $( \pm \mathrm{SD})$ operation time was $266.7 \pm 47.0$ min with a mean $( \pm S D)$ blood loss of
$54.6 \pm 84.1 \mathrm{ml}$. The mean $( \pm \mathrm{SD})$ number of dissected lymph nodes was $30.3 \pm 7.2$. The hepatic branch of the vagus nerve was preserved in 21 patients and the celiac branch of the vagus nerve was preserved in 11 patients. In all patients LAsTG was performed successfully, with no conversion to open surgery. No patient had a cancer-positive pathological margin. The mean $( \pm S D)$ proximal margin size was $2.0 \pm 0.9 \mathrm{~cm}$. The final pathological staging confirmed stage IA gastric cancer in 22 patients, while stage IB gastric cancer was shown in 1 patient.

Four patients $(17.4 \%)$ experienced postoperative complications. Two of these patients underwent reoperation, because of duodenal stump leakage and stenosis of the $\mathrm{Y}$-anastomosis, respectively; the cause of both complications was torsion of the $\mathrm{Y}$-anastomosis. One patient experienced anastomotic bleeding; this was resolved after endoscopic clipping. Three patients (including the previous two with duodenal stump leakage and anastomotic bleeding respectively) had an abdominal abscess and were treated conservatively. The mean times $( \pm S D)$ until first flatus and start of meal intake were $2.4 \pm 0.9$ and $5.0 \pm 2.5$ days, respectively. The mean $( \pm \mathrm{SD})$ postoperative hospital stay was $15.6 \pm 10.7$ days.

All patients were followed up for more than 3 months. No patient suffered heartburn. Two patients (8.7\%) experienced gastrojejunostomy stricture and were treated successfully by endoscopic balloon dilation. Gastrojejunostomy stricture occurred only in the first half of the study period when the gastrojejunostomy was not routinely 


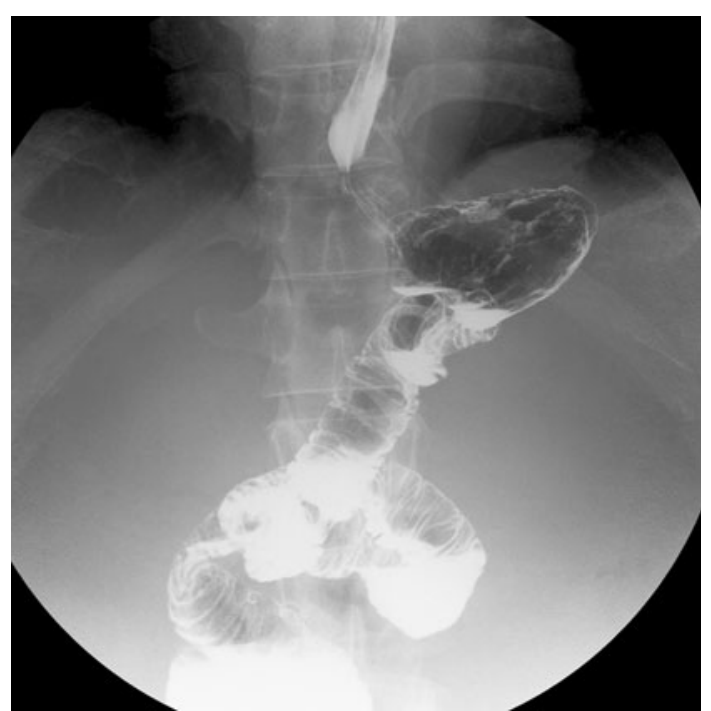

Fig. 5 Barium meal in a patient 9 months after laparoscopy-assisted subtotal gastrectomy (LAsTG) demonstrates a very small remnant stomach with normal transit

checked by gastroscopy during operation. In the second half of the period, after the gastrojejunostomy was checked by gastroscopy in each patient, there were no more occurrences of gastrojejunostomy stricture. Barium meal was performed in 3 patients who underwent LAsTG and showed a very small remnant stomach with normal transit (Fig. 5).

\section{Discussion}

EGC has a very low incidence of local invasion and lymph node metastasis [15] and good long-term survival, so interest has been focused on postoperative quality of life in patients with EGC. LAPG and LATG have been performed as minimally invasive operations for the treatment of EGC in the upper stomach. However, quality of life after proximal gastrectomy or total gastrectomy is still not satisfactory. In some patients with EGC in the upper stomach, there is still some distance from the tumor to the EGJ. Thus, theoretically, it is possible to preserve some stomach, which is beneficial for the postoperative quality of life [16, 17]. There are two major concerns about preserving a very small remnant stomach with surgery for EGC in the upper stomach: one is oncological safety, and the other is the reconstruction technique for gastrojejunostomy with a very small remnant stomach.

In the present study, we report our novel technique for preserving a very small remnant stomach. For this new technique, we always cautiously paid attention to the tumor lesion and lymph node involvement. We confirmed the tumor location and a safe proximal resection margin by placing clips preoperatively and by using intraoperative gastroscopy. The resected proximal margin was sent for frozen pathology if it was suspected of being cancerpositive. According to the Japanese classification of gastric carcinoma, the splenic hilum lymph node (station 10) is regarded as a second tier lymph node for gastric cancer in the upper stomach; however, it is not routinely dissected in our LAsTG procedure. Nevertheless, lymph nodes from the root of the left gastroepiploic vessels (station $4 \mathrm{sb}$ ), which were very near the splenic hilum, and the other second-tier lymph nodes were sent for frozen pathology to confirm that there was no lymph node metastasis. Therefore, the oncological safety of the LAsTG procedure can be assured.

The reconstruction method for gastrojejunostomy is another difficulty with this new technique. Roux-en-Y gastrojejunostomy is the only choice for reconstruction because of the very small remnant stomach. In reports about open or laparoscopic gastrectomy, side-to-side Rouxen-Y gastrojejunostomy was usually performed [18, 19], and this needed a relatively large remnant stomach and sufficient blood supply from the greater curvature of the stomach. However, that kind of technique would not have been suitable for the patients in the present study because the remnant stomach was so small that the blood supply from the short gastric artery would have been interrupted by stapling. Noshiro et al. [11] reported their new technique of making a gastrojejunostomy on the stomach transecting line for a very small remnant stomach, which needed time for suturing the extra incision after gastrojejunostomy. In the present study, we tried to find an easier and faster method for gastrojejunostomy, and we felt that a procedure using a circular-stapling device might be suitable [20, 21]. We applied a modified technique using the $\mathrm{OrVil}^{\mathrm{TM}}$ system and a $25 \mathrm{~mm}$ circular stapler. Such a technique was performed for esophagojejunostomy in the LATG procedure, and it has proved its feasibility [22]. Transoral anvil insertion using the $\mathrm{OrVil}^{\mathrm{TM}}$ system is very simple and time-saving. With this technique, the anvil rod is lodged in the greater curvature of the gastric stump. This is an important aspect of this technique, because it allows safe anastomosis with minimal tension [21]. The final check of the anastomosis by gastroscopy is also necessary to avoid bleeding, leakage, or stenosis. In some centers without substantial experience of LAG, open subtotal gastrectomy can also be performed using our technique, to avoid total gastrectomy for selected patients with EGC in the upper stomach. However, there are some disadvantages related to the $\mathrm{OrVil}^{\mathrm{TM}}$ system. Because the anvil is inserted transorally, this technique is not suitable for patients with stenosis of the pharynx or esophagus. Another concern is potential abdominal contamination caused by a contaminated $\mathrm{OrVil}^{\mathrm{TM}}$ tube. Immediate removal of the $\mathrm{OrVil}^{\mathrm{TM}}$ 
tube after inserting the anvil and careful abdominal irrigation are useful for preventing possible contamination.

Unfortunately, in the present study, two patients experienced reoperation after LAsTG because of duodenal stump leakage and stenosis of the Y-anastomosis, respectively; these are common complications that are not associated with our new technique but are associated with the Roux-en-Y operation. The cause of duodenal stump leakage and stenosis of the Y-anastomosis was torsion of the $\mathrm{Y}$-anastomosis. After strict closure of the mesenterium of the jejunum and Petersen's cavity, the risk of this torsion should be decreased. In any case, to avoid postoperative complications, LAG with Roux-en-Y reconstruction should be performed cautiously by experienced laparoscopic surgeons. Two of our patients experienced gastrojejunostomy stricture, which was related to the new technique. Gastrojejunostomy stricture occurred only in the first half of our study period when the gastrojejunal anastomosis was not routinely checked by gastroscopy during the operation. During the creation of the gastrojejunal anastomosis, not pulling the jejunal limb too tightly is also beneficial for preventing anastomotic stricture. Fortunately, the stricture was not very severe and was resolved by endoscopic balloon dilation. With an increase in experience with this new technique, complications may be decreased.

In summary, we have developed a novel technique, LAsTG, for the treatment of EGC in the upper stomach in selected cases. With the described method, a very small remnant stomach can be preserved. To evaluate the benefit of our new technique, investigations of long-term quality of life in patients treated with LAsTG are warranted.

\section{References}

1. Maehara Y, Kakeji Y, Oda S, Takahashi I, Akazawa K, Sugimachi K. Time trends of surgical treatment and the prognosis for Japanese patients with gastric cancer. $\mathrm{Br} \mathrm{J}$ Cancer. 2000;83:986-91.

2. Hiki N, Shimoyama S, Yamaguchi H, Kubota K, Kaminishi M. Laparoscopy-assisted pylorus-preserving gastrectomy with quality controlled lymph node dissection in gastric cancer operation. J Am Coll Surg. 2006;203:162-9.

3. Kitano S, Shiraishi N, Uyama I, Sugihara K, Tanigawa N. A multicenter study on oncologic outcome of laparoscopic gastrectomy for early cancer in Japan. Ann Surg. 2007;245:68-72.

4. Kim HH, Hyung WJ, Cho GS, Kim MC, Han SU, Kim W, et al. Morbidity and mortality of laparoscopic gastrectomy versus open gastrectomy for gastric cancer: an interim report-a phase III multicenter, prospective, randomized Trial (KLASS Trial). Ann Surg. 2010;251:417-20.

5. Sakuramoto S, Yamashita K, Kikuchi S, Futawatari N, Katada N, Moriya $\mathrm{H}$, et al. Clinical experience of laparoscopy-assisted proximal gastrectomy with Toupet-like partial fundoplication in early gastric cancer for preventing reflux esophagitis. J Am Coll Surg. 2009;209:344-51.
6. Bo T, Zhihong P, Peiwu Y, Feng Q, Ziqiang W, Yan S, et al. General complications following laparoscopic-assisted gastrectomy and analysis of techniques to manage them. Surg Endosc. 2009;23:1860-5

7. An JY, Youn HG, Choi MG, Noh JH, Sohn TS, Kim S. The difficult choice between total and proximal gastrectomy in proximal early gastric cancer. Am J Surg. 2008;196:587-91.

8. Mochiki E, Toyomasu Y, Ogata K, Andoh H, Ohno T, Aihara R, et al. Laparoscopically assisted total gastrectomy with lymph node dissection for upper and middle gastric cancer. Surg Endosc. 2008;22:1997-2002.

9. Jeong GA, Cho GS, Kim HH, Lee HJ, Ryu SW, Song KY. Laparoscopy-assisted total gastrectomy for gastric cancer: a multicenter retrospective analysis. Surgery. 2009;146:469-74.

10. Lee SE, Ryu KW, Nam BH, Lee JH, Kim YW, Yu JS, et al. Technical feasibility and safety of laparoscopy-assisted total gastrectomy in gastric cancer: a comparative study with laparoscopy-assisted distal gastrectomy. J Surg Oncol. 2009;100:392-5.

11. Noshiro H, Ohuchida K, Kawamoto M, Ishikawa M, Uchiyama A, Shimizu $S$, et al. Intraabdominal Roux-en-Y reconstruction with a novel stapling technique after laparoscopic distal gastrectomy. Gastric Cancer. 2009;12:164-9.

12. Tokunaga M, Hiki N, Fukunaga T, Nohara K, Katayama H, Akashi Y, et al. Laparoscopy-assisted distal gastrectomy with D2 lymph node dissection following standardization-a preliminary study. J Gastrointest Surg. 2009;13:1058-63.

13. Kojima K, Yamada H, Inokuchi M, Kawano T, Sugihara K. Functional evaluation after vagus-nerve-sparing laparoscopically assisted distal gastrectomy. Surg Endosc. 2008;22:2003-8.

14. Japanese Gastric Cancer Association. Japanese classification of gastric carcinoma-2nd English edition. Gastric Cancer. 1998;1:10-24.

15. Maruyama K, Kaminishi M, Hayashi K, Isobe Y, Honda I, Katai H, et al. Gastric cancer treated in 1991 in Japan: data analysis of nationwide registry. Gastric Cancer. 2006;9:51-66.

16. Bozzetti F, Marubini E, Bonfanti G, Miceli R, Piano C, Gennari L. Subtotal versus total gastrectomy for gastric cancer: five-year survival rates in a multicenter randomized Italian trial Italian Gastrointestinal Tumor Study Group. Ann Surg. 1999;230:170-8.

17. Jentschura D, Winkler M, Strohmeier N, Rumstadt B, Hagmuller E. Quality-of-life after curative surgery for gastric cancer: a comparison between total gastrectomy and subtotal gastric resection. Hepatogastroenterology. 1997;44:1137-42.

18. Fujita T, Katai H, Morita S, Saka M, Fukagawa T, Sano T. Shortterm outcomes of Roux-en-Y stapled anastomosis after distal gastrectomy for gastric adenocarcinoma. J Gastrointest Surg. 2010;14:289-94.

19. Kojima K, Yamada H, Inokuchi M, Kawano T, Sugihara K. A comparison of Roux-en-Y and Billroth-I reconstruction after laparoscopy-assisted distal gastrectomy. Ann Surg. 2008; 247:962-7.

20. Gonzalez R, Lin E, Venkatesh KR, Bowers SP, Smith CD. Gastrojejunostomy during laparoscopic gastric bypass: analysis of 3 techniques. Arch Surg. 2003;138:181-4.

21. Omori T, Oyama T, Akamatsu H, Tori M, Ueshima S, Nakahara $\mathrm{M}$, et al. A simple and safe method for gastrojejunostomy in laparoscopic distal gastrectomy using the hemidouble-stapling technique: efficient purse-string stapling technique. Dig Surg. 2010;26:441-5.

22. Jeong O, Park YK. Intracorporeal circular stapling esophagojejunostomy using the transorally inserted anvil (OrVil) after laparoscopic total gastrectomy. Surg Endosc. 2009;23:2624-30. 